\title{
FLUID-STRUCTURE ANALYSIS FOR THE HDR BLOWDOWN AND SNAPBACK EXPERIMENTS WITH FLUX
}

\author{
A. LUDWIG and U. SCHUMANN \\ Kernforschungszentrum Karlsruhe GmbH, Institut für Reaktorentwicklung, Projekt Nukleare Sicherheit, Postfach 3640, D-7500 \\ Karlsruhe, Fed. Rep. Germany \\ Received January 1982
}

FLUX is a special purpose code to analyse three-dimensional fluid-structure interactions during blowdown of a pressurized water reactor. Such a blowdown has been simulated in the HDR experiments. For the first series of blowdown experiments and for snapback experiments in the same facility the results of precomputations are reported and compared with the experimental results. Refinements are desirable with respect to two-phase-damping of pressure waves in the blowdown pipe and vessel wall flexibility. The general quantitative agreement between measurement and computation is satisfactory.

\section{Introduction}

A postulated sudden break of a pressurized water reactor (PWR) coolant-pipe causes a rapid depressurization and blowdown of the reactor vessel water content. The resultant transient pressure field imposes large forces on the vessel's internal structures. The structures have to resist these loadings. The analysis of such events is complicated by fluid-structure interaction, i.e. the feedback mechanism by which the fluid motion excites structural motion which in turn changes the fluid motion [1,2]. Several computer codes have been developed to analyse such motions; overviews have been given in $[3,4]$. To check the validity of these codes, blowdown and snapback experiments have been performed at the HDR test-facility $[5,1]$. In this paper we report comparisons between the experimental results and computations done with the code FLUX (version FLUX2 together with the shell model CYLDY 3 and the two-phase model DRIX-2D).

The geometric arrangement for the tests is shown in fig. 1. In the HDR experiment, the internal vessel structures of a PWR are represented by an idealized core barrel. This barrel is a cylindrical shell clamped to a rather rigid flange at the upper edge and connected with a stiff mass ring, which serves to simulate roughly the mass of a PWR core, at the lower edge.

The blowdown is initiated by a membrane break at the end of the blowdown pipe attached to nozzle A1. Fig. 2 illustrates the resultant motion field. Two experi- ments, V29.2 and V31, are considered which differ mainly with respect to the length of the blowdown pipe, see table 1. We also include data for case V31.1 which replicated V31 in order to check for the reproducibility of the experiments. In the snapback experiments the core barrel is deflected quasi-statically by some deflec-

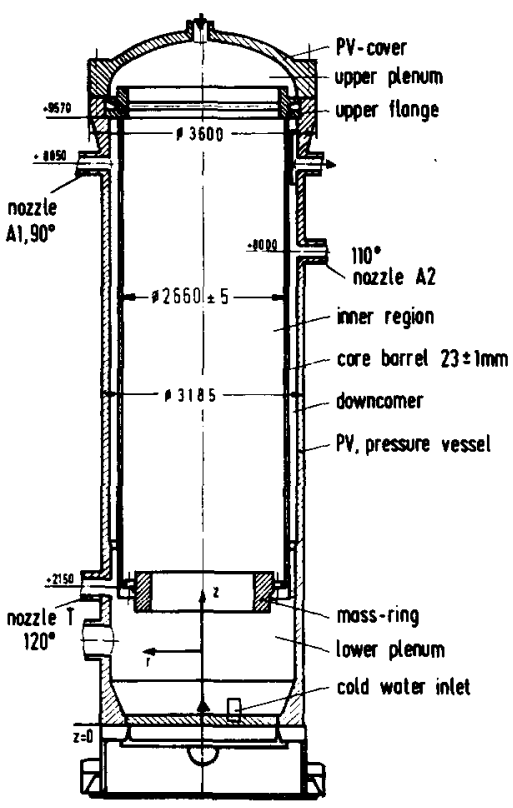

Fig. 1. The HDR vessel with core barrel. 


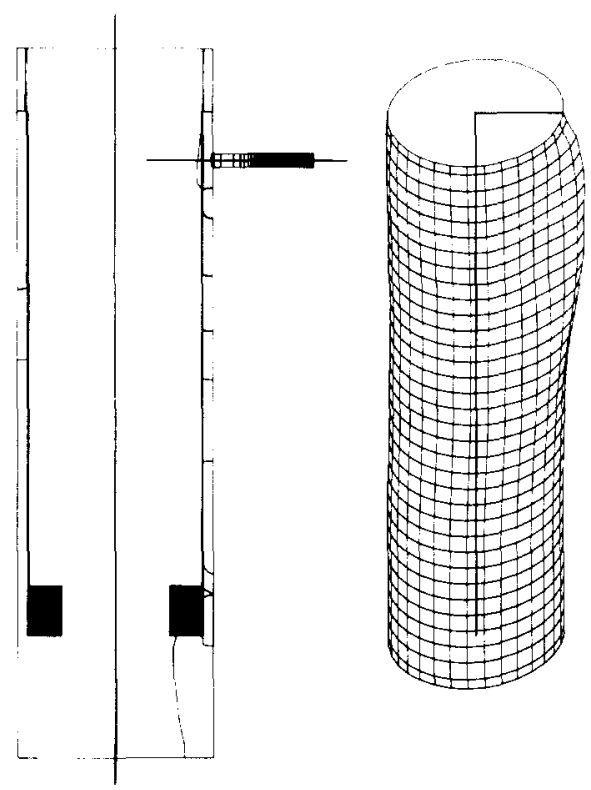

Fig. 2. Computed isobars ( 1 bar spacing) and core barrel deflection (300 times magnified) for $\mathrm{V} 31$ at $t=10 \mathrm{~ms}$. tion devices and then suddenly released. The loading is introduced either at the lower core barrel edge where it is stiffened by the mass ring ("nozzle T") or at a shell location $1.57 \mathrm{~m}$ below the upper flange ("nozzle A2"); here the loading is distributed on a plate of $0.3 \times 0.3$ $\mathrm{m}^{2}$. Fig. 3 shows the computed initial deflection of the core barrel.

The code FLUX ("FLU id in fle $\boldsymbol{X}$ ible structure") [6] is a special purpose code. It is designed to analyse the PWR blowdown loadings during the initial period of a blowdown while most of the water remains in its liquid state (subcooled water). Snapback and other external forces (one could consider seismic forces, e.g.) can be included. Several code versions have been developed. FLUX1 assumes incompressible fluid and treats fluidstructure coupling by means of added masses [7]. For the HDR we use FLUX2 which treats compressible, potential flow. It provides an implicit time-integration scheme which is applied simultaneously to the fluid and the structure. A version FLUX3 [8] has been developed which allows for treatment of nonlinearities due to impacts at supports with gaps, but this version is not

Table 1

Experimental parameters as used for the precomputations

\begin{tabular}{lllcc}
\hline Blowdown & & & & \\
\hline Parameter & Symbol & Unit & Cases & V31 \\
& & & V29.2 & 110 \\
\hline Initial pressure & $p_{0}$ & bar & 90 & 268 \\
$\begin{array}{l}\text { Initial temperature in } \\
\text { the blowdown pipe }\end{array}$ & $T_{0}$ & & & 52 \\
Saturation pressure & $p_{\text {sat }}$ & bar & 271 & 1.50 \\
Pipe length & $L_{\mathrm{S}}$ & $\mathrm{m}$ & 4.66 & 2 \\
Break-opening time & $t_{\mathrm{B}}$ & $\mathrm{ms}$ & 2 & \\
\hline
\end{tabular}

\section{Snapback}

\begin{tabular}{|c|c|c|c|c|c|c|}
\hline \multirow[t]{2}{*}{ Parameter } & \multirow[t]{2}{*}{ Symbol } & \multirow[t]{2}{*}{ Unit } & \multicolumn{4}{|c|}{ Cases V59 } \\
\hline & & & 1 & 2 & 3 & 4 \\
\hline Loading at nozzle & - & - & $\mathrm{T}$ & $\mathrm{T}$ & A2 & $\mathrm{A} 2$ \\
\hline Fluid & - & - & air & water & air & water \\
\hline Initial deflection & $w_{0}$ & $\mathrm{~mm}$ & 3.5 & 3.5 & 2 & 2 \\
\hline Initial pressure & $p_{0}$ & bar & 1 & 20 & 1 & 20 \\
\hline Computed load force & $F_{0}$ & MN & -0.65 & -0.65 & 0.139 & 0.139 \\
\hline
\end{tabular}



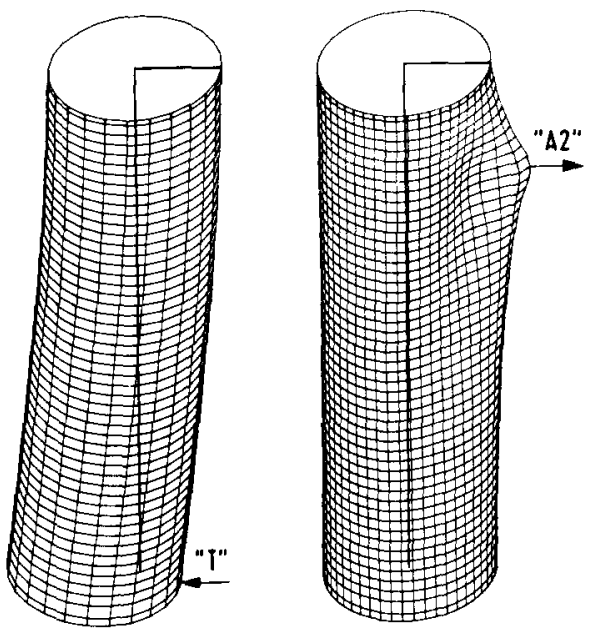

Fig. 3. Computed initial deflection of the core barrel for the snapback experiments V59.1 and V59.2 (left, 200 times magnified) and V59.3 and V59.4 (right, 300 times magnified).

relevant for the HDR experiment. At present a model for the reactor core is being incorporated in FLUX [9]. FLUX requires that a model for the dynamics of the core barrel and the pressure vessel be provided. For the present computations, the shell model CYLDY3 [10] is used for this purpose, while the vessel has been taken as being rigid. Alternatively we have used the shell model CYLDY2 [11]. The fluid flow in the blowdown pipe is covered either directly with FLUX2 or treated separately with the two-phase non-equilibrium model DRIX-2D [12]. For the present purpose all these code components are comprised under the heading FLUX. All code components are programmed in PL/ 1 .

In the following, the basic models are summarized; thereafter the specific HDR parameters are defined and then the comparison with the experiments is reported.

\section{The FLUX2 model}

\subsection{Nomenclature}

a constant speed of sound

$c$ vector of generalized structure coordinates

$E=\frac{1}{2} \rho_{0} u^{2}$, pressure head

$F$ force per unit area

$n$ normal on interface

$p$ pressure field

$p_{0}$ initial pressure

$p_{F}$ pressure at the break p vector of discrete pressure values

$t$ time

$u \quad$ fluid velocity

$w$ shell deflection

$\boldsymbol{x}$ space coordinate

$\beta=1 / 4$, Newmark parameter

$\kappa$ constant friction coefficient

$\rho$ density

$\rho_{0} \quad$ initial density

$\Phi \quad$ velocity potential

(dot represents local time derivative)

\subsection{Fluid model}

The assumptions used are:

- potential flow:

$\boldsymbol{u}=\operatorname{grad} \Phi$

- linear (acoustic) equation of state

$p-p_{0}=a^{2}\left(\rho-\rho_{0}\right) ;$

- friction force per unit mass $\sim \rho_{0} \kappa u$;

- small Mach numbers, $(\boldsymbol{u} / a)^{2} \ll \mathrm{I}$.

With these assumptions Euler's equation states

$\rho_{0} \dot{\boldsymbol{u}}=-\operatorname{grad}(p+E)-\rho_{0} \kappa \boldsymbol{u}$,

and the continuity equation is

$\dot{\rho}=-\operatorname{div}\left(\rho_{0} \boldsymbol{u}\right)$.

From the time derivative of eq. (4) and with eqs. (2) and (3) one obtains a wave equation for the fluid pressure:

$(\ddot{p}+\kappa \dot{p}) / a^{2}-\operatorname{div} \operatorname{grad}(p+E)=0$,

and a Laplace equation for the potential

$\operatorname{div} \operatorname{grad}\left(\rho_{0} \dot{\Phi}+\rho_{0} \kappa \Phi+p+E\right)=0$.

\subsection{Structural model}

The assumptions are:

- linear-elastic core barrel shell and rigid walls otherwise (in the next series of computations the vessel will be considered to be elastic),

- axisymmetric core barrel geometry so that the shell eigensolutions are harmonic functions of the azimuthal angle,

- linear ansatz $w(x, t)=v(c(t), x)$ for the shell deflection $w$ in terms of generalized coordinates $c=\left\{c_{i}\right\}$; the ansatz can be a series expansion in smooth functions as in the CYLDY2-model [11], a series expansion in the eigensolutions as in the CYLD3-model 
[10] or a finite difference or finite element ansatz which keeps the symmetry property,

- the structural model provides the mass-, dampingand stiffness matrices in

$\boldsymbol{M c}+\boldsymbol{D} \dot{c}+\boldsymbol{S c}=\boldsymbol{q} \equiv\left\{q_{i}\right\}$,

$q_{i}=\oint F \cdot\left(\partial v / \partial c_{i}\right) \mathrm{d} O$

The integral is taken over the fluid--structure interface.

\subsection{Fluid-structure interface and boundary conditions}

The assumptions are:

- the force imposed by the fluid on the structure interface is $\boldsymbol{F}=-p \boldsymbol{n}$,

- structural deflections ( $w$ ) are small in comparison to the downcomer width so that the interface boundary condition for the fluid can be prescribed at the undeformed wall.

From continuity and eq. (3) follows

$\boldsymbol{n} \cdot \dot{\boldsymbol{w}}=\boldsymbol{n} \cdot \boldsymbol{u}=\boldsymbol{n} \cdot \operatorname{grad} \boldsymbol{\Phi}$,

$\rho_{0} n \cdot \ddot{w}=-n \cdot \operatorname{grad}(p+E)-\rho_{0} \kappa \boldsymbol{n} \cdot \boldsymbol{u}$

at the interface. At openings the pressure $p_{\mathrm{F}}(t)$ is prescribed and the boundary condition for $\boldsymbol{n} \cdot \operatorname{grad} \Phi$ follows from eq. (3). As a further assumption $n \cdot \operatorname{grad} E$ is taken as zero at the opening surface.

\subsection{Space discretization}

In planes through the vessel axis, finite differences are used. In the azimuthal coordinate, the pressure field is expanded in a finite Fourier series. The blowdownpipe is approximated one-dimensionally with finite differences. The resultant set of equations can be imagined as written in matrix form

$$
\begin{aligned}
& {\left[\begin{array}{ll}
M & \\
-V & K
\end{array}\right]\left\{\begin{array}{l}
\ddot{\boldsymbol{c}} \\
\ddot{\boldsymbol{p}}
\end{array}\right\}+\left[\begin{array}{ll}
D & \\
& D_{\mathrm{F}}
\end{array}\right]\left\{\begin{array}{l}
\dot{\boldsymbol{c}} \\
\dot{\boldsymbol{p}}
\end{array}\right\}+\left[\begin{array}{ll}
S & R \\
& L
\end{array}\right]\left\{\begin{array}{l}
\boldsymbol{c} \\
\boldsymbol{p}
\end{array}\right\}} \\
& =\left\{\begin{array}{l}
\boldsymbol{\theta} \\
f\left(p_{\mathrm{F}}(t), E(t)\right)
\end{array}\right\} \equiv \boldsymbol{r}(t) .
\end{aligned}
$$

Here, $\boldsymbol{M}, \boldsymbol{D}$, and $\boldsymbol{S}$ are given by the structural model, see eq. (7). $K, D_{\mathrm{F}}, L$, and $f$ appear from the spatial discretization of the wave equation (5). $K$ and $D_{F}$ are diagonal matrices, $\boldsymbol{L}$ is the discretizated Laplace operator in the fluid domain. The pressure loading on the structure is represented by $\boldsymbol{R}$ and $\boldsymbol{V} \sim \boldsymbol{R}^{\mathrm{T}}$ is a consequence of the coupling condition (10).
The Laplace equation (6) with boundary condition (9) has the simple solution

$\rho_{0} \dot{\phi}+\rho_{0} \kappa \phi+p+E=\psi(t)$.

The parameter $\psi(t)$ can be selected so that $\phi=0$ for an arbitrary point $\boldsymbol{x}_{\mathrm{s}}$ in the fluid.

\subsection{Time discretization}

Let us write eq. (11) as $\boldsymbol{M}^{\prime} \ddot{\boldsymbol{s}}+\boldsymbol{D}^{\prime} \boldsymbol{s}+\boldsymbol{S}^{\prime} \boldsymbol{s}=\boldsymbol{r}$, with $s^{\mathrm{T}}=\left(\boldsymbol{c}^{\mathrm{T}}, \boldsymbol{p}^{\mathrm{T}}\right)$. The Newmark- $\beta$-scheme is used for time discretization:

$$
\begin{aligned}
\boldsymbol{M}^{\prime}( & \left.\delta s_{n+1}-\delta s_{n}\right) / \Delta t^{2}+D^{\prime}\left(\delta s_{n+1}+\delta s_{n}\right) /(2 \Delta t) \\
& +\boldsymbol{S}^{\prime}\left[s_{n}+\beta\left(\delta s_{n+1}-\delta s_{n}\right)\right] \\
= & r_{n}+\beta\left(\delta r_{n+1}-\delta r_{n}\right) \\
s_{n+1} & =s_{n}+\delta s_{n+1} .
\end{aligned}
$$

Here, $s_{n}$ approximates $s(n \Delta t)$. The nonlinear pressure head is included in $\boldsymbol{r}_{n}=\boldsymbol{r}(n \Delta t)$ but not in $\delta \boldsymbol{r}_{n+1}=\boldsymbol{r}_{n+1}$ $-\boldsymbol{r}_{n}$ in order to make the system linear in the unknowns $s_{n+1}$ at the new time level. With $\beta=1 / 4$ the scheme is unconditionally stable for the linear part, second order accurate and numerically non-damping. No instabilities due to the non-linear term have been found as long as the fluid does not flow over more than one grid cell per time step. At each time step a linear system of the form

$$
\begin{aligned}
& {\left[\begin{array}{ll}
\boldsymbol{M} / \Delta t^{2}+\boldsymbol{D} /(2 \Delta t)+\beta \boldsymbol{S} & \beta \boldsymbol{R} \\
-\boldsymbol{V} / \Delta t^{2} & \boldsymbol{K} / \Delta t^{2}+\boldsymbol{D}_{\mathbf{F}} /(2 \Delta t)+\beta \boldsymbol{L}
\end{array}\right]} \\
& \left\{\begin{array}{l}
\boldsymbol{\delta} \boldsymbol{c}_{n+1} \\
\boldsymbol{\delta} \boldsymbol{p}_{n+1}
\end{array}\right\}=\boldsymbol{r}_{n}^{\prime}
\end{aligned}
$$

is solved using fast elliptic solvers, which are special efficient methods [6]. Thereafter the new solutions at time level $n+1$ are obtained from eq. (14). The new discrete potential values $\boldsymbol{\phi}_{n+1}$ are obtained from a discrete representation of eq. (12) which is consistent with eqs. (13) and (14):

$$
\begin{aligned}
\boldsymbol{\Phi}_{n+1}= & \left\{(1-\Delta t \kappa / 2) \dot{\Phi_{n}}+\Delta t \psi_{n} / \rho_{0}-\left(\Delta t / \rho_{0}\right)\right. \\
& \left.\times\left[p_{n}+\beta\left(\delta p_{n+1}-\delta p_{n}\right)+E_{n}\right]\right\} /(1+\Delta t \kappa / 2) .
\end{aligned}
$$

From the new potential values the new velocities, see eq. (1), and the pressure head $\boldsymbol{E}_{n+1}$ are determined. 


\section{The model parameters for the HDR cases}

\subsection{Experimental cases}

The experimental setup is described in detail in [13]. We have performed precomputations for the whole set of experiments, i.e. the blowdown experiments V29.2 and V31, and the snapback experiments V59.1 to V59.4. Characteristic data, as specified for the precomputations, are given in tables I and 2. We should note that the data of the actual experiments show some discrepancies [5]. However, these are not essential to explain differences between experiment and computation.

\subsection{Structural model}

The core barrel shell is modeled by CYLDY3 [10], i.e. by a finite set of eigensolutions of Flüge's shell equations. Fig. 4 shows the eigenfrequencies as a function of the azimuthal Fourier mode in vacuum. All modes of these eigenfrequencies below about $1000 \mathrm{~Hz}$ up to the 16th azimuthal order are included in the discrete model. This amounts to 423 degrees of freedom for the core barrel.

\subsection{Fluid model}

Fig. 5 shows the geometrical model. Because of symmetry only one half of the vessel is treated. In the experiment a piping system is connected with the lower half of the vessel [13]; these pipes are not represented in the computation. Model parameters are the fluid density $\rho_{0}$, the speed of sound a and the friction coefficient $\kappa$. The density is determined according to the initial conditions in the blowdown pipe. For the speed of sound the relation

$a=\left[\left(p_{0}-p_{\text {sat }}\right) /\left(\rho_{0}-\rho_{\text {sat }}\right)\right]^{1 / 2}$

is taken where $\left(p_{0}, \rho_{0}\right)$ correspond to the initial state

Table 2

Material data of the core barrel for blowdown (snapback)

\begin{tabular}{lll}
\hline Symbol & Parameter & Data \\
\hline$\rho_{\mathrm{M}}$ & Density & $7900 \mathrm{~kg} / \mathrm{m}^{3}$ \\
$E$ & Young's modulus & $1.75(1.98) \times 10^{11} \mathrm{~N} / \mathrm{m}^{2}$ \\
$\nu$ & Poisson's ratio & $0.295(0.28)$ \\
$M_{\mathrm{R}}$ & Mass of the ring & $13517 \mathrm{~kg}$ \\
$I_{\mathrm{ZZ}}$ & $\begin{array}{l}\text { Rotational inertia } \\
\text { of ring }\end{array}$ & $7086.3 \mathrm{~kg} \mathrm{~m}$ \\
\hline
\end{tabular}

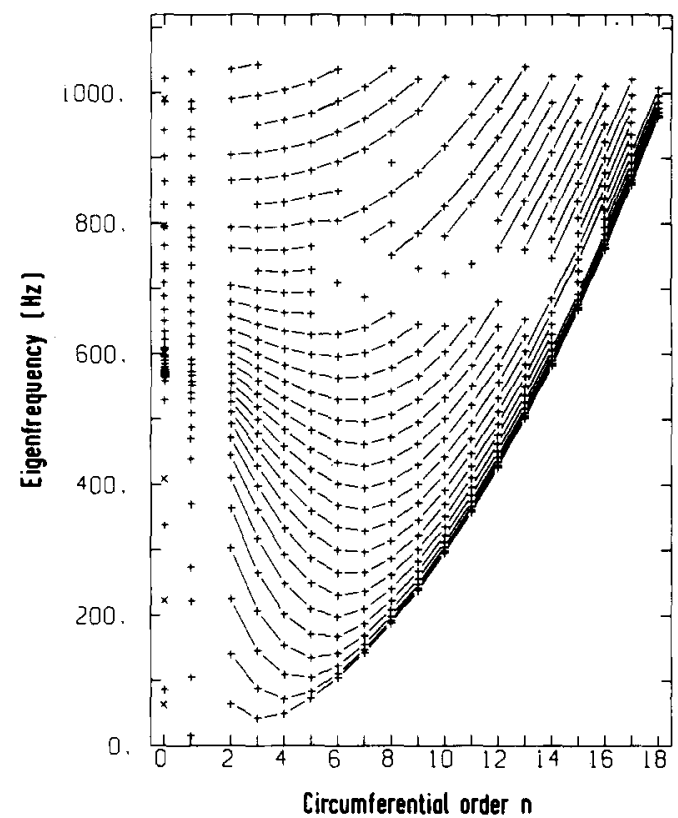

Fig. 4. Computed eigenfrequencies of the core barrel in vacuum versus azimuthal Fourier mode $n$.

and $\left(p_{\text {sat }}, \rho_{\text {sat }}\right)$ to the liquid saturated state at the same entropy. In the code, the speed of sound can be defined separately for each of the five domains indicated in

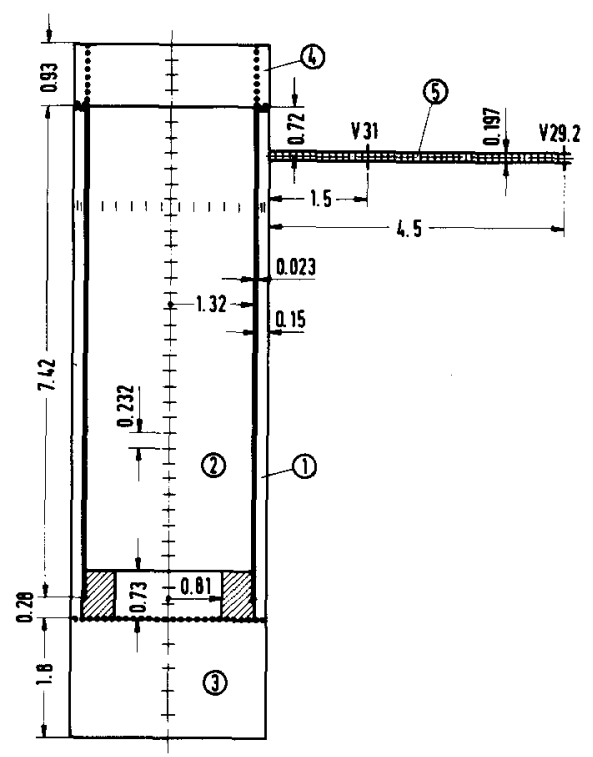

Fig. 5. FLUX model with geometrical dimensions for V29.2 and V31. The grid spacing is indicated on the axis, on one radius, and in the blowdown pipe. 
fig. 5. Generally this possibility is used to account for different initial temperatures. In the present case we use modified sound speeds in the domains 1 and 5 to account for the flexibility of the pressure vessel wall (thickness $\approx 0.112 \mathrm{~m}$ ) and the blowdown pipe wall (thickness $\approx 0.01 \mathrm{~m}$ ) using standard formulae [2]. This results in a reduction of $23 \%$ in the downcomer and $4 \%$ in the pipe. The finally resultant effective sound speed will be even smaller due to the flexibility of the core barrel, but this effect is automatically accounted for by the fluid-structure coupling algorithm. In the future the vessel will be treated in the same manner; then the nominal sound speed will be used as input value for the downcomer.

The friction coefficient $k$ is determined from

$\kappa=f_{\text {damp }} u_{\max } / 4 R_{\mathrm{s}}, \quad u_{\max }=\left[2\left(p_{0}-p_{\mathrm{sat}}\right) / \rho_{0}\right]^{1 / 2}$

as explained in [14]. The non-dimensional friction coefficient $f_{\mathrm{damp}}$ is set to 0.02 . In case of the snapback-experiments fluid friction is set to zero, $\kappa=0$. For structural damping $3 \%$ modal damping [6, eq. (15)] is used in all cases.

With respect to the blowdown pipe, two approaches have been used. In the first (case "FX2"), FLUX2 is used to model the whole blowdown pipe. At the exit the pressure is prescribed to be

$p_{\mathrm{F}}(t)= \begin{cases}p_{0}+\left(p_{\mathrm{sat}}-p_{0}\right) \sin ^{2}\left(\pi t / 2 t_{\mathrm{B}}\right) & \text { for } t \leqslant t_{\mathrm{B}} \\ p_{\mathrm{sat}} & \text { for } t>t_{\mathrm{B}}\end{cases}$

A preestimated "break time" $t_{\mathrm{B}}=2 \mathrm{~ms}$ is being used. In the experiments an even faster pressure drop has been found. The value $t_{\mathrm{B}}=1.3 \mathrm{~ms}$ would give the best fit. From parameter studies [6] we conclude that the difference in the break times has negligible effects on all results except for the accelerations.

In the second approach (case "FXD"), FLUX2 is coupled with DRIX-2D [12] in order to describe the two-phase non-equilibrium effects in the blowdown pipe more realistically. DRIX-2D uses a two-dimensional axisymmetric model which is well suited for the blowdown pipe and the outer area into which the water is blown. The geometry of the vessel, however, cannot be correctly simulated. As indicated in fig. 6 , only the vessel volume and the downcomer width are modeled.

One way of coupling between DRIX-2D and FLUX (called "weak" coupling) consists of taking the pressure (averaged over the pipe cross-section) from DRIX-2D $0.11 \mathrm{~m}$ downstream the nozzle as a boundary value for a subsequent FLUX2 computation. This approach does

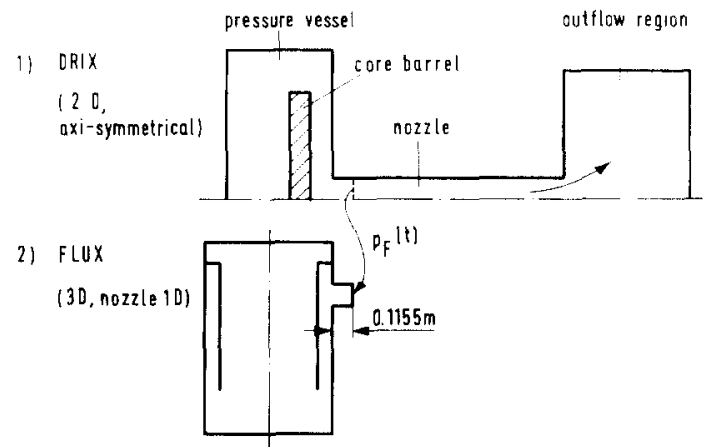

Fig. 6. The "weak" coupling scheme of DRIX-2D with FLUX2.

not guarantee that the mass fluxes across this surface in FLUX2 and DRIX-2D are always equal. In fact, they differ slightly.

Therefore, Enderle [15] has performed a "strong" coupling which enforces mass, momentum and energy conservation at the coupling surface for each time step. The strong coupling treats FLUX and DRIX-2D as two independent computer-tasks which are synchronized after each time step using the PL/1 multitasking facilities.

The strong coupling generally gives results with are closer to the experimental data, but with respect to the core barrel motion the differences are only marginal, see fig. 7 . On the other hand, the strong coupling requires an order of magnitude larger computing times.

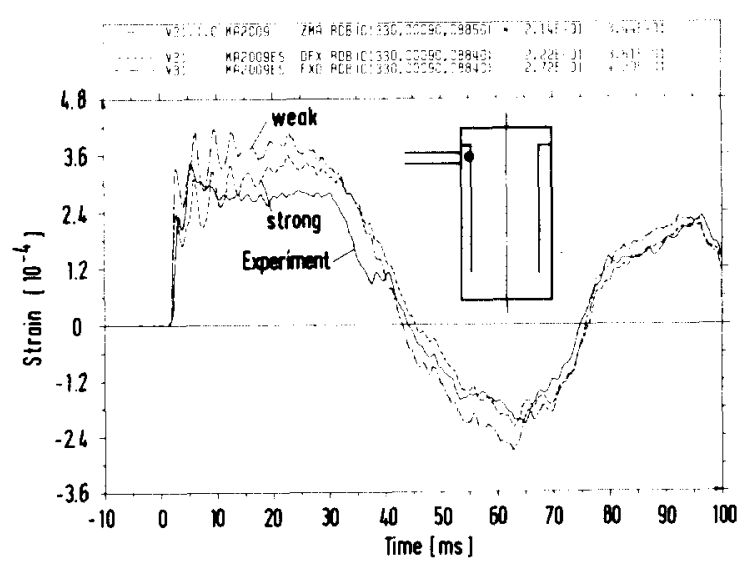

Fig. 7. Azimuthal strain in the core barrel near the blowdown nozzle versus time. Comparison between measurement (ZMA), weakly coupled DRIX-FLUX computation (FXD) and strongly coupled DRIX-FLUX computation (DFX). 
All subsequent "FXD"-results are from the weak coupling procedure.

The spatial discretization of the fluid domain in FLUX2 comprises 9 radial cells in the vessel, 15 in the pipe (V31), 42 axial cells and 16 azimuthal intervals from 0 to $180^{\circ}$. The discretization is indicated in fig. 5 . The total number of mesh cells is 5754 . The time step for both the fluid and the structure is $0.2 \mathrm{~ms}$. This discretization is close to that of grid N3 discussed in [6]. Parameter studies [6] have shown that this grid is fine enough to get reliable pressure differences and core barrel deflections. A total of $100 \mathrm{~ms}$ has been simulated. The computing time of FLUX2 was $5040 \mathrm{~s}$ on an IBM 3033. In the meantime this time has been reduced to $3600 \mathrm{~s}$ by minor code changes.

In the DRIX-2D computations a total of 205 grid cells were used $(19 \times 5$ in the pipe). The time step size was $0.02 \mathrm{~ms}$ and the computing time on an IBM 3033 was $9900 \mathrm{~s}$ (the code has now been made faster by a factor of about 2.5).

\section{Discussion of the blowdown results}

\subsection{Overview}

A general description of the blowdown experimental results and comparison with several code results has been given in [5]. It has been shown that the experimental results are very well reproducible and generally of sufficient accuracy to be taken as benchmarks for the codes. The differences between prespecified and actual test parameters are marginal. We had no incentive to perform postcomputations, therefore. For case V31 many code results are available. It has been found that the FLUX2-DRIX combination did perform very successfully. The general agreement is of about the same quality as for the other multi-dimensional codes (K-FIX [16], FLEXWALL [17]) inspite of the physical simplifications used for the flow model in the vessel. For case V29.2 results of other multidimensional fluid-structure analysis codes are not existing. For this case, with a rather long pipe, it has been found that the acoustical fluid model of FLUX2 does not match the measured behaviour in the blowdown pipe [18] as will be explained below. For this reason, V31 has been run with the FLUX2/DRIX combination.

\subsection{Pressure in the blowdown pipe}

Fig. 8 shows the measured pressure in the mid of the blowdown pipe in comparison to computation with

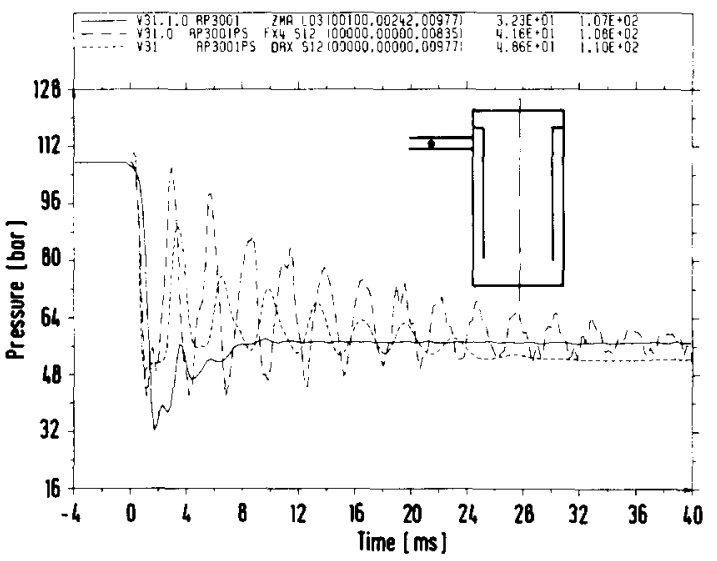

Fig. 8. Pressure in the blowdown test V31, measurement (ZMA), acoustical model (FX4), FLUX/DRIX-2D model (FXD). In the figure legend the transducer coordinates and the minimum and maximum signal values for this time period are given.

FLUX2 (i.e. the acoustical model) and DRIX (i.e. the nonequilibrium two-phase model).

In the acoustical pipe-model pressure waves are hardly damped. In fact the only physical damping mechanism included in this model are the friction damping in terms of the parameter $\kappa$ in eq. (3) and the radiation of wave energy from the pipe into the vessel. At the pipe exit the pressure is kept constant; this means that no energy is radiated outwards. In a specific parameter study [19] we have also used an acoustical flow model (with constant sound speed) which included the outflow domain so that radiation becomes possible. However, the results have shown that this radiation effect is far too small to explain the differences to the measurements. The same type of acoustic waves had been found for case V29.2 [18]. As a consequence of these waves, the pressure averaged over a period of one wave period $(\approx 10 \mathrm{~ms}$ in $\mathrm{V} 29.2, \approx 2.5 \mathrm{~ms}$ in $\mathrm{V} 31)$ does not drop to the saturation pressure. This means that the effective pressure difference which causes the loads in the vessel is smaller $(\approx 50 \%$ in V29.2) so that this model underestimates the core barrel deflections etc. Corresponding figures have been shown in [18].

It is very interesting, however, that also the DRIX results show considerable pressure oscillations. They are damped faster than in the acoustical model but not as fast as in the experiment. This result is not completely understood because DRIX models the phase change (with corresponding changes in the speed of sound, which causes dispersion), and it contains slip (internal friction) and a delayed evaporation model (which should 
cause damping). It does not contain a model for thermal non-equilibrium (different phase temperatures) and may, hence, overpredict the potential for recondensation due to lacking entropy increase. However due to recent studies with K-FIX ' this should have minor effects.

Some codes which are based on purely homogeneous equilibrium models like FLUST [20] and MULTIFLEX [21] do predict the correct wave damping. However, these models describe isentropic flow. Thus, there is no physical reason for damping in these models. We believe that the damping encountered in these codes is essentially of numerical nature.

Obviously, a convincing quantitative explanation of the physical mechanism which causes the damping is yet missing. One might note that none of the codes used for the HDR case includes a model for nucleation. From the analysis of Alamgir \& Lienhard [22] one can conclude that a large part of the pressure undershoot is due to delayed evaporation which can be modeled in terms of a nucleation model.

It seems very likely that all these discussions are relevant only for the extremely short break opening times considered here. Larger break opening times $t_{\mathrm{B}}$ are realistically assumed in nuclear reactor safety analysis (typically $15 \mathrm{~ms}$ ). If the ratio $t_{\mathrm{B}} a / L_{\mathrm{S}}(a=$ speed of sound, $L_{\mathrm{S}}=$ pipe length) becomes larger than one, then virtually no pressure waves are excited in the pipe. This number has the value 0.09 for case V29.2 and 0.29 for V31.1 and indeed the errors due to the pressure waves are smaller in the latter case.

\subsection{Pressure in the downcomer}

Fig. 9 shows that the pressure in the downcomer is matched in its general trend. In the first $20 \mathrm{~ms}$ some aftermath of the not sufficiently damped waves can be seen in the numerical result. At later times the computed pressure drops too fast. This is partly caused by a somewhat too large mass flux out of the blowdown pipe and partly due to an underestimate of the change in vessel volume due to the elasticity of the vessel wall during blowdown. The pressure difference at the core barrel, fig. 10, shows typical fluid-structure interaction effects. The small positive spike in the first $10 \mathrm{~ms}$ is due to outward acceleration of the core barrel. The relatively small pressure wave speed (as compared to the computation for rigid core barrel) originates from the added compressibility [2] induced by the elasticity of the core barrel shell.

\footnotetext{
Information presented in connection with [16].
}

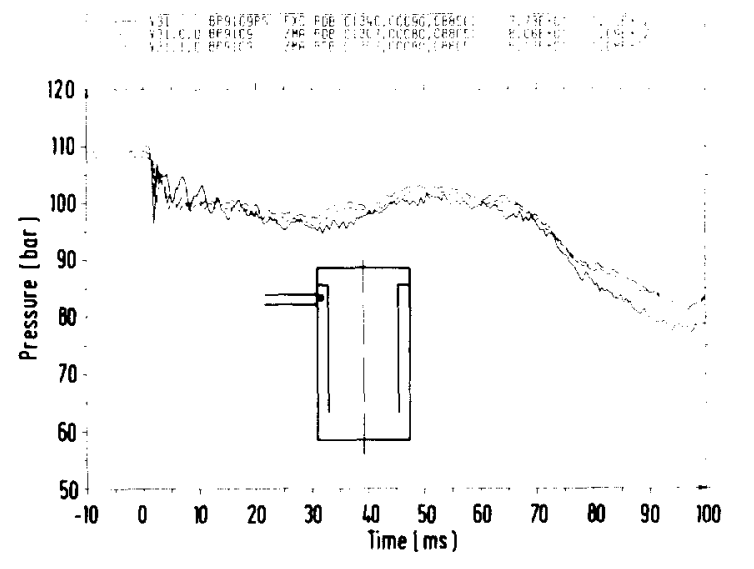

Fig. 9. Pressure in the downcomer near the nozzle. Comparison of two measurements (ZMA) with FLUX (FXD).

\subsection{Deflection and strain in the core barrel}

Fig. 11 shows a comparison with respect to the radial deflection of the core barrel. It should be noted that the transducers measure the relative displacement between vessel and core barrel. The vessel motion which was not included in these precomputations might be responsible for some of the discrepancies. (The results found by Takeuchi [21] do not rule out this explanation because he included the beam mode motion of the vessel only: the displacement measurements at the vessel also show significant shell mode motions.) Of course, also some model errors might be the reason for the differences. However, this is not very likely because the measured

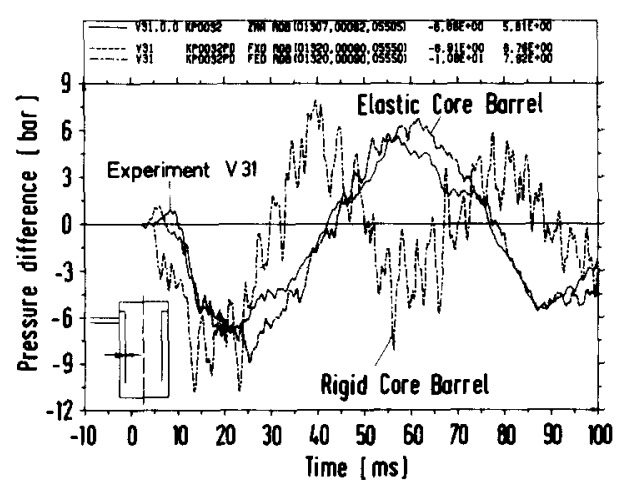

Fig. 10. Pressure differences across the core barrel wall for blowdown. Comparison between measurement (ZMA), FLUX/DRIX-2D model with elastic core barrel (FXD) and same model with rigid core barrel (FED). 


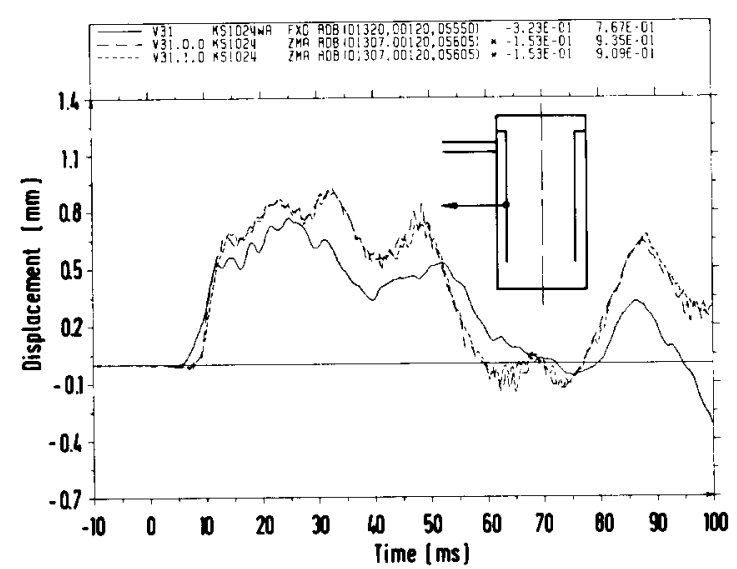

Fig. 11. Core barrel deflection below the nozzle for blowdown. Comparison of two measurements (ZMA) with FLUX (FXD).

strains at the core barrel, see fig. 12 , are matched very well. This is true at least for the azimuthal strains measured at $180^{\circ}$ with respect to the blowdown nozzle. Near the blowdown nozzle the computations overestimated the strains and show large oscillations, see fig. 7 . These may again be due to the pressure waves in the pipe but can also be caused by a Gibb's phenomenon. The latter effect is known to cause oscillating approximations to curves with large local changes by an insufficient number of Fourier modes. Such local changes are peculiar to the near nozzle domain.

We note that the largest deflections and strains occur after about $30 \mathrm{~ms}$. In this time period and considerably

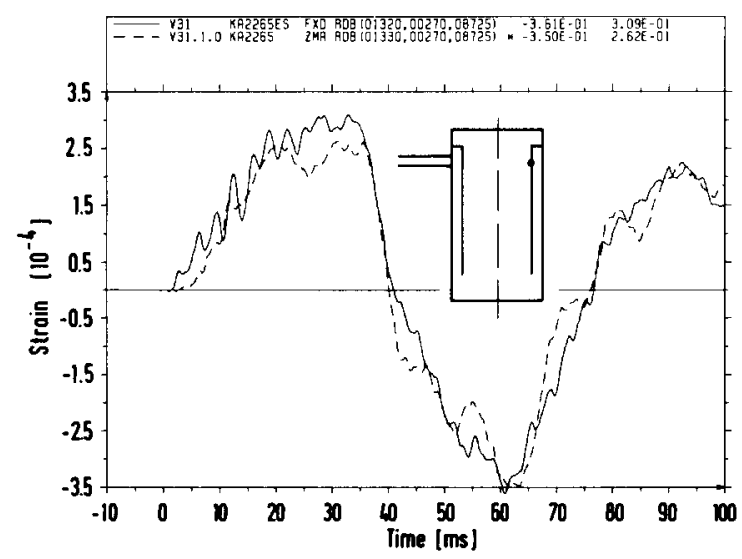

Fig. 12. Azimuthal strain in the core barrel at a location opposite to the nozzle for blowdown. Comparison of measurement (ZMA) with FLUX (FXD). beyond (up to about $120 \mathrm{~ms}$ ) the water remains in its subcooled state as assumed in the FLUX model. In fact thermodynamic nonequilibrium effects in the vessel interiour allow the pressure to drop below the saturation pressure without immediate evaporation. This effect extends the period where the model is valid. Further we see that the displacement amplitudes are of the order of $1 \mathrm{~mm}$ or $1 \%$ of the downcomer width. Theoretical analysis shows that the change in virtual masses, e.g., is quadratic in the relative displacement, such that the linear fluid-structure coupling model is justified.

\section{Discussion of the snapback results}

\subsection{Overview}

The HDR-snapback test series V59 has been designed to be a separate check of the structure dynamics model as well as of the fluid-structure interaction models without two-phase flow phenomena imposed. The experimental setup and some results have been reported in [23]. A complete comparison between experiments and precomputations by all participants has been compiled in [24]. The experiments in air are a check for the pure structural model. As shown in [25] the air mass is negligible for all structural modes except for the breathing mode, but this mode is excited only weakly. Both in air and water the system behaves such that linear models are appropriate for the first $100 \mathrm{~ms}$ (on a time scale of $10 \mathrm{~s}$, excitation of a higher shell mode has been observed [23] which can be explained in terms of nonlinear fluid-structure coupling).

\subsection{Eigenfrequencies and modes}

From the experimental data, eigenfrequencies and corresponding modes have been identified using a special analysis code EVA $[23,16]$. Numerically, eigensolutions in vacuum are provided directly by the shell model CYLDY3. Eigenoscillations in air or water have been computed for incompressible fluid using FLUX1. For the low frequency eigensolutions, neglection of compressibility is justified $[25,30]$.

As shown in fig. 13, computed eigenfrequencies compare generally well with the experimental results. This holds both for the pressure vessel filled with air or water. Some frequencies of low vibration orders, however, are calculated too high in both cases. These differences are likely due to the finite flexibility of the mass ring, the upper flange and the vessel. 

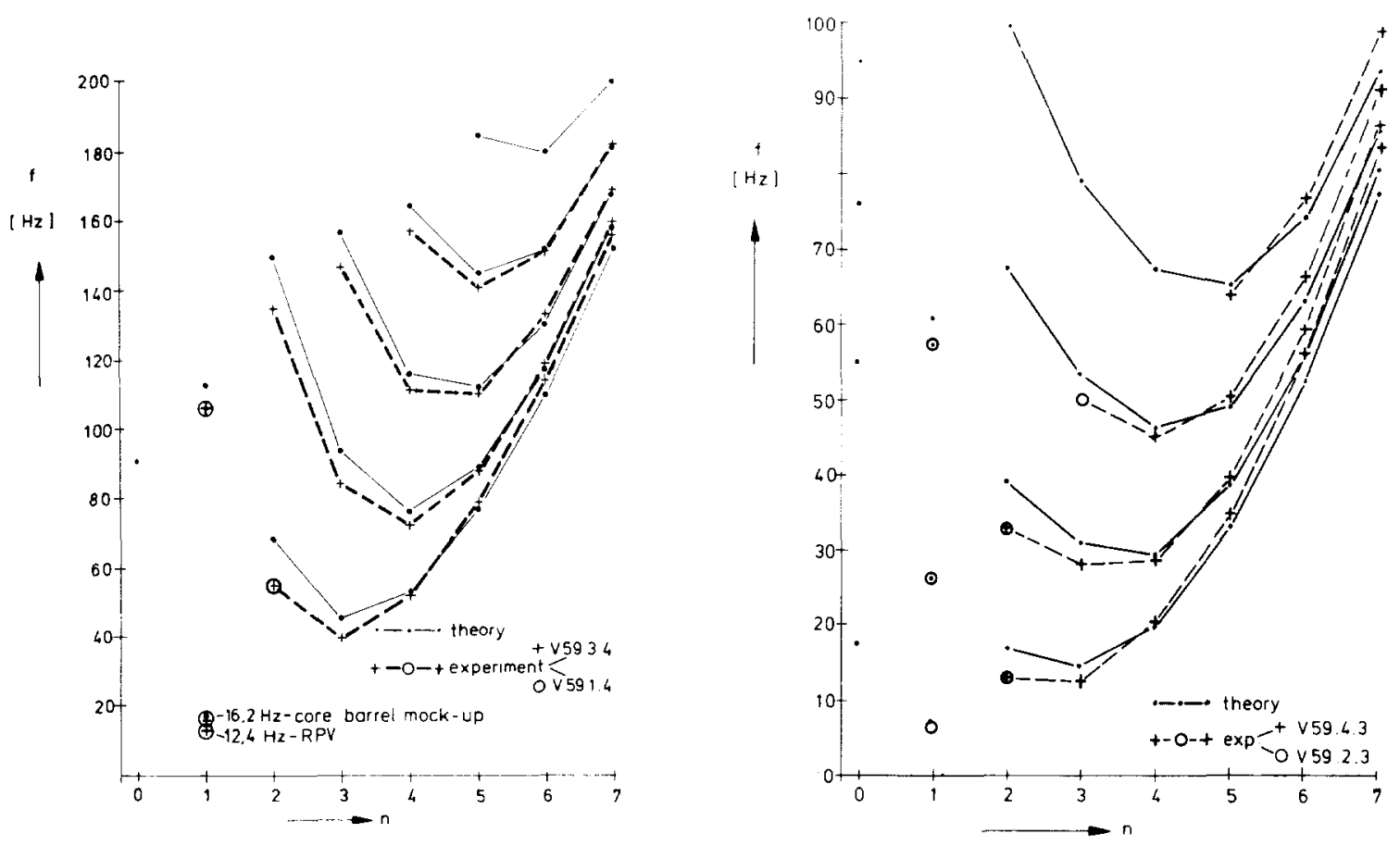

Fig. 13. Comparison between calculated and measured eigenfrequencies (left: in air, right: in water).

\subsection{Damping}

In the experiments, structural and fluid damping have been found to be very small $(0.1$ to $1.6 \%$ [26]). One can show, using the theories of Yeh \& Chen [27] that laminar viscous damping results in a modal damping coefficient which is of the order $S^{-1 / 2}$ for $S \gg 1$ where $S$ is the Strouhal number

$S=\omega L^{2} / \nu$,

( $\omega=$ characteristic angular frequency, $L=$ characteristic length scale, e.g. the downcomer width $H_{\mathrm{R}}, \nu=$ kinematic viscosity). The typical Strouhal number is at least of the order $10^{6}$ which explains the small fluid damping found. The preestimated structural damping of $3 \%$ is generally an order of magnitude too large.

\subsection{Time histories}

Transient simulations have been done using FLUX2, i.e. the compressible fluid option. Figs. 14-16 show a set of typical results. Unfortunately the initial state deflection did not always coincide with the prespecifications which had been used for code input. This explains some of the differences in amplitude. All computed results compare favorably with the measured signals at least with respect to the low frequency content (up to $200 \mathrm{~Hz}$ ). After $40 \mathrm{~ms}$, however, the correlations become weaker. This holds for all four snapback cases, i.e. in water and air for excitations at the lower edge and in the shell domain.

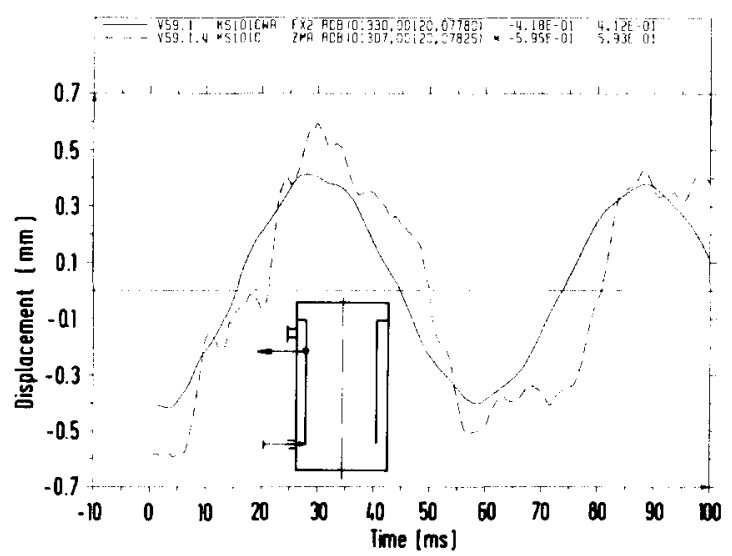

Fig. 14. Core barrel deflection in the upper region for snapback V59.1 (excitation at the lower end; RPV filled with air). Comparison of measurement (ZMA) with FLUX (FX2). 


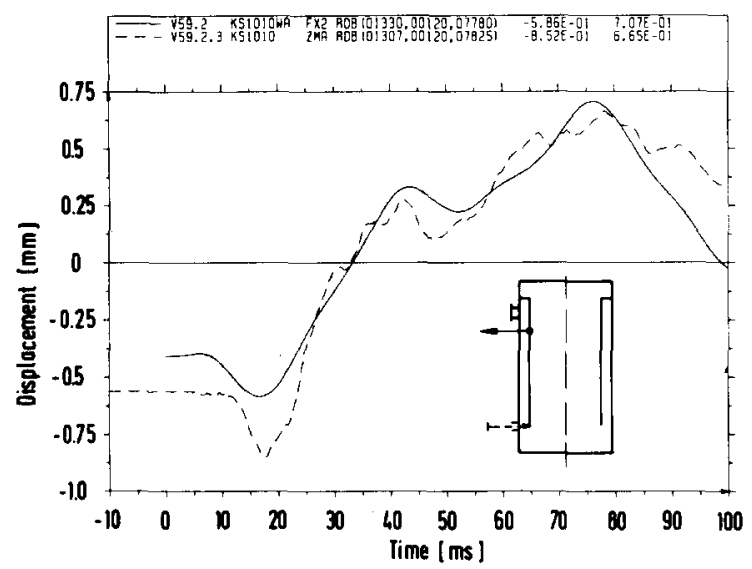

Fig. 15. Core barrel deflection in the upper region for snapback V59.2 (excitation at the lower end; RPV filled with water). Comparison of measurement (ZMA) with FLUX (FX2).

Larger differences appear for the high frequency components. These differences are particularly obvious from acceleration and pressure signals. Some of these differences can be attributed to unsufficient resolution. The highest eigenfrequency considered in the shell model is $1000 \mathrm{~Hz}$. Thus, higher frequencies cannot be resolved. Other reasons might stem from the break behavior of the deflection devices and the flexibility of the mass ring. Anyway, these high frequency differences are of small relevance for nuclear reactor safety analysis.

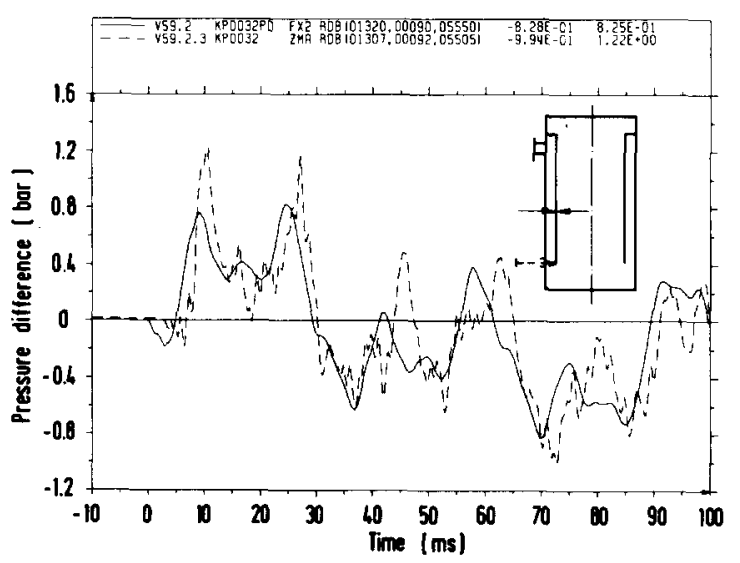

Fig. 16. Pressure difference across core barrel wall for snapback V59.2 (exciation at the lower end; RPV filled with water). Comparison of measurement (ZMA) with FLUX (FX2).

\section{Conclusions}

Results have been reported of the FLUX computer code for the HDR blowdown and snapback experiments. The results have been precomputed before the experiments had been performed. In view of the experimental results, there was little incentive to perform a post-test calculation for three reasons: (1) the initial values matched fairly well those used for the predictive calculations, (2) the results were satisfactory in most essential aspects, and (3) the FLUX code is basically built on first principles and contains almost no empirical coefficient to adjust.

The comparison has shown that fluid-structure interaction has to be taken into account for realistic analysis. Although the HDR experiment overemphasizes this effect, this conclusion should be transferred to real PWR analysis as several parameter studies have shown [28-30].

The general quantitative agreement between measurement and computation is such that it should be sufficient for safety analysis.

For short break times the fluid model should be able to describe the observed strong pressure wave damping in the blowdown pipe. A purely acoustical model predicts unrealistic slowly decaying pressure waves which result in an underestimation of the loads on the core barrel. Obviously, the two phase flow causes this damping, however the actual mechanism is not yet explained. The non-equilibrium two-phase code DRIX gives better but still not ideal results. Nevertheless, the FLUX2/DRIX-combination (in its weak form) is satisfying even for short break times. DRIX fairly well predicts the pressure undershoot in the first $10 \mathrm{~ms}$ near the break.

The snapback experiments have shown that the CYLDY3/FLUX2 combination gives close approximations for the measured lowest eigenfrequencies and modes in air and stagnant water. Some of the lower modes are apparently affected by the vessel motion.

All these findings support the validity of the assumptions used. In particular, single phase irrotational fluid flow with constant speed of sound is appropriate for the vessel interior and for the time period during which the largest loadings on the core barrel appear. Also the fluid-structure coupling algorithm which is restricted to pressure loads and small interface deflections has been confirmed.

The FLUX2 code requires an order of one hour IBM 3033 computing time for each of the cases considered here (up to $100 \mathrm{~ms}$ problem time). This computing time is such that parameter studies become not prohibitively 
expensive. For short break times, the coupling with DRIX becomes necessary; this makes the analysis more expensive.

Improvements are possible. For example, a higher resolution might improve the acceleration and strain results, in particular those for positions near the nozzle where strong local curvatures arise. Moreover, we are now prepared to analyse the next series of experiments with a shell model for the vessel motion itself, which has been found to have significant size. Further, we are experimenting with a simple scheme to model the wave damping effects within the FLUX2 model.

On a whole. one can say that this type of problem can be satisfactorily analyzed with the existing tools.

\section{Acknowledgement}

Several colleagues have contributed to this work. In particular we mention the following: Mr. F. Katz performed the DRIX-2D calculations. Dr. G. Enderle implemented the strong coupling of DRIX-2D and FLUX and provided results. Mr. H. Knüppel produced the computer plots. Mr. F. Eberle and Mr. J. Kadlec determined the modal results from the snapback measurements. Dr. E.G. Schlechtendahl contributed several important discussions. Finally we are grateful for the excellent cooperation with the HDR project team.

\section{References}

[1] R. Krieg, E.G. Schlechtendahl and K.-H. Scholl, Design of the HDR experimental program on blowdown loading and dynamic response of PWR-vessel internals, Nucl. Engrg. Des. 43 (1977) 419-435.

[2] U. Schumann, Principles of fluid-structure interactions, in: Proc. Intern. Topical Meetg. on Advances in Mathematical Methods for the Solution of Nuclear Engineering Problems, München, April 27-29, 1981 (Fachinformationszentrum Energie, Physik, Mathematik, Karlsruhe, 1981), Vol. I, pp. 565-582.

[3] E.G. Schlechtendahl, Status of code-development in the Federal Republic of Germany concerning fluid-structural coupling during reactor transients, Nucl. Safety 20 (1979) $551-563$.

[4] T. Belytschko and U. Schumann, Fluid-structure interactions in light water reactor systems, Nucl. Engrg. Des. 60 (1980) 173-195.

[5] L. Wolf, U. Schumann and K.-H. Scholl, Experimental and analytical results of coupled fluid-structure interactions during blowdown of the HDR-vessel, in: Proc. 6th Int. Conf. on Structural Mechanics in Reactor Technology, Paris, Aug. 1981, paper B2/1.
[6] U. Schumann, Fast elliptic solvers and three-dimensional fluid-structure interactions in a pressurized water reactor. J. Comp. Phys. 36 (1980) 93-127.

[7] U. Schumann. Dynamics of a nuclear-reactor shell-structure in an incompressible fluid, in: Recent Developments in Theoretical and Experimental Fluid Mechanics, eds. U. Müller et al. (Springer, Berlin, 1979), pp. 577-586.

[8] U. Schumann, Impacts and fluid-structure interactions in pressurized water reactor safety analysis. Nucl. Engrg. Des. 69 (1982) 313.

[9] U. Schumann and J. Benner. Homogenized model for fluid-structure interactions in a pressurized water reactor core, in: Proc. 6th Internat. Conf. on Structural Mechanics in Reactor Technology, Paris, Aug. 1981, paper B5/7.

[10] A. Ludwig and R. Krieg, An analytical quasi-exact method for calculating eigenvibrations of thin circular cylindrical shells, J. Sound Vibr. 74 (1981) 155-174.

[11] A. Ludwig and R. Krieg, Dynamic response of a clamped/ring-stiffened circular cylindrical shell under non-axisymmetric loading, Nucl. Engrg. Des. 43 (1977) 437-453.

[12] H. Mösinger, Investigation of blowdown problems with the computer code DRIX-2D, Nucl. Sci. Engrg. 76 (1980) 89- 102.

[13] L. Wolf (ed.), Preliminary design report for the RPV-I blowdown experiments V29, V30 and V31 with specifications for the pretest computations, PHDR-Report No. 3/151/79, HDR Sicherheitsprogramm, Kernforschungszentrum Karlsruhe (1979).

[14] U. Schumann, Analysis of the RS16B experiment on fluid-structure interactions during PWR-blowdown, in: Proc. 5th Internat. Conf, on Structural Mechanics in Reactor Technology, Berlin, Aug. 1979, paper B6/4.

[15] G. Enderle, private communication (Jan. 1981).

[16] C.W. Hirt, W.C. Rivard and M.D. Torrey, Post test evaluaton of K-FIX computations for a full scale HDR blowdown test. LA-UR 81-1645, 2nd Internat. Seminar on Fluid-Structure Interaction and Internal Loading in Thermal Reactors, ISPRA, Italy, Aug. 24-25, 1981.

[17] R. Hummel, P. Gruber and W. Rott, Influence of fluidshell interaction on load and displacement time histories using the FLEXWALL computer code for HDR pretest calculations and comparison thereof with experimental results, in: Proc. 6th Internat. Conf. on Structural Mechanics in Reactor Technology, Paris, Aug. 1981, paper B2/3.

[18] U. Schumann, A. Badur, P. Gruber and L. Wolf, Ergebnisse der ersten Blowdown-Versuche mit KernmantelVergleich der Messungen mit Vorausrechnungen, in: 4.Statusbericht des Projektes HDR, PHDR-Arbeitsbericht 05.3/80, HDR-Sicherheitsprogramm, Kernforschungszentrum Karlsruhe (1980), pp. 7-0-7-38.

[19] U. Schumann, Uberprüfung der Auswirkung der Druckrandbedingung an der Bruchstelle auf die Wellenausbreitung beim Blowdown mit dem Programm WELLE2. (1980) unpublished.

[20] G. Enderle, Compressible flow in arbitrarily connected fluid domains, in: Proc. 3rd GAMM-Conf. Numerical 
Methods in Fluid Mech., ed. E.H. Hirschel, (F. Vieweg, Braunschweig, 1980) pp. 88-97.

[21] K. Takeuchi, Method of characteristics for fluid-structure interactions and analysis of HDR blowdown experiments, 2nd Internat. Seminar on Fluid-Structure Interaction and Internal Loading in Thermal Reactors, ISPRA, Italy, Aug. 24-25, 1981.

[22] M. Alamgir and J.H. Lienhard, Correlation of pressure undershoot during hot-water depressurization, J. Heat Transfer, Trans. ASME 103 (1981) 52-55.

[23] F. Eberle, E. Erb, J. Kadlec, P. Philipp and E. Wolf, HDR snapback experiments, in: Proc. 6th Internat. Conf. on Structural Mechanics in Reactor Technology, Paris, Aug. 1981, paper B3/1.

[24] A. Ludwig and M. Geiss, Eigenschwingversuche ("Snapback-Versuche") am HDR-Kernmantel, Quick Look Report RDB-E V59, Report PHDR 22-81, HDRSicherheitsprogramm, Kernforschungszentrum Karlsruhe (1981).

[25] A. Ludwig and U. Schumann, Calculations on the HDR core-barrel response during snap-back tests, in: Proc. 5th Internat. Conf. on Structural Mechanics in Reactor Technology, Berlin, Aug. 1979, paper B6/7.
[26] F. Eberle and J. Kadlec, Erfahrungen aus der modalanalytischen Auswertung von Snapback-Versuchen, in: Wind- und erdbebenerregte Schwingungen von Bauwerken, VDI-Bericht Nr. 419 (VDI-Verlag, Düsseldorf, 1981) pp. 39-45.

[27] T.T. Yeh and S.S. Chen, The effect of fluid viscosity on coupled tube/fluid vibrations, J. Sound Vibr. 59 (1978) 453-467.

[28] U. Schumann, Fluid-Struktur-Wechselwirkung des Kernmantels und der Brennstäbe beim Kühlmittelverluststörfall, Atomkernenergie/Kerntechnik 36 (1980) $305-$ 310.

[29] V.F. Koch and W. Pohl, Numerical analysis of 3-D pressure-wave propagation and -loading of the core barrel of a PWR caused by a LOCA, in: Proc. 6th Internat. Conf. on Structural Mechanics in Reactor Technology, Paris, Aug. 1981, paper B2/8.

[30] R. Krieg and U. Schumann, Zur Kompressibilität und virtuellen Masse bei Blowdown-Beanspruchungen von Reaktordruckbehältereinbauten, Atomkernenergie/Kerntechnik 39 (1981) 57-63. 